\title{
Dimensionless Analysis of HSDM and Application to Simulation of Breakthrough Curves of Highly Adsorbent Porous Media
}

\author{
Agustí Pérez-Foguet, M.ASCE ${ }^{1}$; Eva Casoni ${ }^{2}$; and Antonio Huerta, M.ASCE ${ }^{3}$
}

\begin{abstract}
The homogeneous surface diffusion model (HSDM) is widely used for adsorption modeling of aqueous solutions. The Biot number is usually used to characterize model behavior. However, some limitations of this characterization have been reported recently, and the Stanton number has been proposed as a complement to be considered. In this work, a detailed dimensionless analysis of HSDM is presented and limit behaviors of the model are characterized, confirming but extending previous results. An accurate and efficient numerical solver is used for these purposes. The intraparticle diffusion equation is reduced to a system of two ordinary differential equations, the transport-reaction equation is discretized by using a discontinuous Galerkin method, and the overall system evolution is integrated with a time-marching scheme. This approach facilitates the simulation of HSDM with a wide range of dimensionless numbers and with a correct treatment of shocks, which appear with nonlinear adsorption isotherms and with large Biot numbers and small surface diffusivity modulus. The approach is applied to simulate the breakthrough curves of granular ferric hydroxide. Published experimental data is adequately simulated.
\end{abstract}

CE Database subject headings: Adsorption; Reactors; Porous media; Simulation.

Author keywords: Adsorption modeling; Plug-flow fixed-bed reactor; Intraparticle mass profile; Biot number; Stanton number; Discontinuous Galerkin.

\section{Introduction}

The homogeneous surface diffusion model (HSDM) has been used extensively for adsorption modeling processes in porous media. It is a dual-resistance model that includes the influence on adsorption of film mass transfer (of the adsorbate diluted in interstitial fluid into the adsorbent particles) and of intraparticle diffusion (of the solid-phase adsorbate inside the adsorbent particles). A general description of HSDM and various practical applications, in particular those related to wastewater treatment can be found, for example, in the work of Weber and Smith $(1986,1987)$ and Brusseau and Gillham (1989). Other adsorption models as the homogeneous pore diffusion model (HPDM) or the heterogeneous pore-surface diffusion model (PSDM) share main characteristics with HSDM, see

${ }^{1}$ Associate Professor, Laboratori de Càlcul Numèric, Dept. de Matemàtica Aplicada III, Institut de Sostenibilitat, E.T.S d'Enginyers de Camins, Canals i Ports de Barcelona, Universitat Politècnica de Catalunya-BarcelonaTech (UPC), Edifici C2, Campus Nord UPC, E-08034 Barcelona, Spain (corresponding author). E-mail: agusti .perez@upc.edu

${ }^{2}$ Postdoctorate, Laboratori de Càlcul Numèric, Dept. de Matemàtica Aplicada III, E.T.S d'Enginyers de Camins, Canals i Ports de Barcelona, Universitat Politècnica de Catalunya-BarcelonaTech (UPC), Edifici C2, Campus Nord UPC, E-08034 Barcelona, Spain.

${ }^{3}$ Professor, Laboratori de Càlcul Numèric, Dept. de Matemàtica Aplicada III, E.T.S d'Enginyers de Camins, Canals i Ports de Barcelona, Universitat Politècnica de Catalunya-BarcelonaTech (UPC), Edifici C2, Campus Nord UPC, E-08034 Barcelona, Spain.
Crittenden et al. (1987), Zhang and Ritter (1997) and Zeng et al. (2008) for detailed presentations.

The HSDM behavior has been characterized in the Biot number, $\mathrm{Bi}$, by several authors (Lee et al. 1983; Hand et al. 1983, 1984; Traegner and Suidan 1989b). The Biot number is the ratio between the film mass-transfer rate and the intraparticle surface diffusion. The literature describes three regions in which the relative importance of the two processes is different. In one region, $\mathrm{Bi} \ll 10^{0}$, film mass transfer dominates, and in another, $\mathrm{Bi} \gg 10^{2}$, surface diffusion dominates. In between those two regions, both processes have a significant influence on the results. These limits have been referenced in several papers dealing with completely mixed flow problems (Hand et al. 1983; Traegner and Suidan 1989a, b), which, in turn, have been referenced in subsequent studies (Roy et al. 1993; Flora et al. 1998; Baup et al. 2000; Chang et al. 2004; Badruzzaman et al. 2004). More recently, similar limits have been referenced in the characterization of generic adsorbents using shallow-bed reactors (Sonetaka et al. 2009a, b). References to these, or similar limits, have been less frequent in works dealing with HSDM plug-flow problems (Lee et al. 1983; Hand et al. 1984; Brattebo and Odegaard 1986; Sperlich et al. 2005, 2008; Genz et al. 2008), although HSDM is also widely used in problems of this sort (Oimstead and Weber 1990; Smith 1996; Rahman et al. 2003). Other works have also used Bi to characterize HSDM results (Smith 1997; Wolborska 1999).

Despite that HSDM has become popular, the behavior of this model has not yet been completely characterized. Further analysis of the relationships between the limit behaviors of the model and the values of key dimensionless numbers is required. Model behavior description based only on the $\mathrm{Bi}$ is not enough. The need to complement the limits of $\mathrm{Bi}$ has been explicitly highlighted by Sperlich et al. (2008) in a study that simulated breakthrough curves (BTCs), that is, the concentration outlet of a plug-flow fixed-bed 
test with granular ferric hydroxide (GFH) using the HSDM. The Stanton number, St, which is the ratio between film mass-transfer rate and flow velocity, was proposed as a complement to $\mathrm{Bi}$ in the characterization of HSDM results.

Here, a dimensionless analysis of HSDM is presented, with the aim of confirming influences of dimensionless numbers on BTCs. Results are obtained with an efficient new HSDM solver that can adequately simulate shocks, such as those found with nonlinear isotherms and with large values of $\mathrm{Bi}$ and small values of the surface diffusivity modulus, Ed. The intraparticle diffusion equation is reduced to a system of two ordinary differential equations (ODEs) by using a Galerkin approximation with two polynomials as base functions. This system of ODEs is coupled with the partial differential equation representing transport-reaction, which is discretized spatially with a discontinuous Galerkin scheme (Sherwin et al, 2006; Casoni et al. 2013). The overall system is time-integrated with the standard forward Euler method. The method is able to simulate the evolution of either smooth or steep concentration profiles without affecting stability.

An outline of the paper follows. The dimensionless numbers and the mathematical model are presented in "Mathematical Model." Key points of the numerical solver are summarized in "Numerical Solver." In "Results", the influence of dimensionless numbers on BTCs is assessed and the limit behaviors of HSDM are characterized. In "GFH Characterization with HSDM," the benefits of the presented approach are demonstrated by simulating GFH BTCs, previously analyzed in the literature (Sperlich et al. 2008). The paper ends with the main conclusions.

\section{Mathematical Model}

The HSDM involves two partial differential equations that represent physical processes at two different scales: the macroscale, which is a porous media with a fluid in movement throughout a matrix of adsorbent particles; and the microscale, which represents the adsorbent particles, assumed to be spherical and also porous themselves. The velocity field of the macroscale flow is considered given. Flow and adsorption are considered uncoupled, a hypothesis that is equivalent to considering the fluid density constant.

The first partial differential equation is an unsteady transportreaction equation that depends on the macroscale coordinates. It takes into account convection, diffusion, and adsorption of components diluted in the interparticle fluid into the adsorbent particles. The second partial differential equation is an unsteady diffusion equation, expressed in the radius of a spherical microscale particle. Both partial differential equations are evolving in time and hence, time-dependent. The intraparticle diffusion equation takes into account the fluid-solid phase change and the solid diffusion of adsorbed mass inside the particles. Isothermal equilibrium between solid and fluid phases is considered, with a functional relationship between diluted and adsorbed mass. Both partial differential equations are coupled through the adsorption term of the transportreaction equation and the external boundary condition of the intraparticle diffusion equation.

The problem can be expressed in a dimensionless form by introducing the dimensionless variables $t=t^{\prime} V / L, x=x^{\prime} / L$, and the field $v=v^{\prime} / V$, with $L$ and $V$ as the reference values of length and velocity, and $t^{\prime}, \boldsymbol{x}^{\prime}$, and $\boldsymbol{v}^{\prime}$ equal to, time, standard spatial coordinates and flow velocity in the macroscale porous media, respectively. The unknowns are $c(\boldsymbol{x}, t)=c^{\prime}\left(\boldsymbol{x}^{\prime}, t^{\prime}\right) / c_{\text {ref }}$ and $q_{P}(\boldsymbol{x}, t)=q_{P}^{\prime}\left(\boldsymbol{x}^{\prime}, t^{\prime}\right) / q_{\mathrm{ref}}$, where $c^{\prime}$ and $q_{P}^{\prime}$ are equal to concentration of adsorbate in porous media fluid and the mean value of adsorbed mass in the adsorbent particles, respectively. The parameters $c_{\text {ref }}$ and $q_{\text {ref }}$ are the corresponding reference values of both unknowns.

The dimensionless numbers of the model are the following:

$$
\begin{aligned}
& \mathrm{Pe}=\frac{V L}{D}, \quad r_{\varepsilon p}=\frac{1-\varepsilon f}{\varepsilon f} \varepsilon_{p}, \quad D_{g}=\frac{r_{\varepsilon_{p}}}{\varepsilon_{p}} \frac{q_{\mathrm{ref}} \rho_{p}}{c_{\mathrm{ref}}}, \\
& \mathrm{Bi}=\frac{r_{\varepsilon p}}{\varepsilon p} \frac{k_{f} R}{D_{g} D_{s}}, \quad \mathrm{St}=\frac{r_{\varepsilon_{p}}}{\varepsilon_{p}} \frac{k_{f}}{R} \frac{L}{V}, \\
& \mathrm{Ed}=\frac{D_{g} D_{S}}{R^{2}} \frac{L}{V}=\frac{\mathrm{St}}{\mathrm{Bi}}, \quad L\left(q_{P}\right)=\frac{q_{P^{1 / n}}}{A^{1 / n}}, \quad A=A^{\prime} \frac{c_{\mathrm{ref}}^{n}}{q_{\mathrm{ref}}}
\end{aligned}
$$

where $\mathrm{Pe}=$ Peclet number; $D=$ interparticle diffusion; $r_{\varepsilon_{p}}=$ porosity ratio; $\varepsilon f$ and $\varepsilon_{p}=$ the inter- and intraparticle porosities; $D_{g}=$ solute distribution parameter (defined as the ratio of the adsorbed mass and the interparticle diluted mass, in steady-state conditions and equilibrium); $\rho_{p}=$ density of clean particles; $k_{f}=$ film mass-transfer coefficient; $R=$ particle radius; $D_{S}=$ intraparticle surface diffusion; and $L\left(q_{P}\right)=$ Freundlich isotherm function, which depends on the parameters $A^{\prime}$ and $n$.

With this notation in mind, the dimensionless macroscale equation can be written as

$$
\frac{\partial c}{\partial t}=\nabla \cdot\left(\frac{\nabla c}{\mathrm{Pe}}\right)-\boldsymbol{v} \cdot \nabla c-\left[D_{g}+r_{\varepsilon_{p}} \frac{\partial L\left(q_{P}\right)}{\partial q_{P}}\right] \frac{\partial q_{P}}{\partial t}
$$

where $\nabla=$ gradient with respect to $x$. Highly adsorbent media are characterized by large values of $D_{g}$. In such cases, the second part of reaction term can be neglected (that proportional to $r_{\varepsilon_{p}}$, which represents mass diluted in intraparticle porosity) hence, simplifying Eq. (2).

The dimensionless intraparticle diffusion equation is given in radial coordinates and can be expressed as

$$
D_{g} \frac{\partial q}{\partial t}=\operatorname{Ed} \frac{1}{r^{2}} \frac{\partial}{\partial r}\left(r^{2} \frac{\partial q}{\partial r}\right)
$$

where $q(r, t ; \boldsymbol{x})=$ dimensionless adsorbed mass profile inside the particle, which depends on $r$ and $t$ for a given $x$. Eq. (3) is complemented by symmetry and Robin-type boundary conditions for the center of the particle and for the particle surface, respectively

$$
\left.\frac{\partial q}{\partial r}\right|_{r=0}=0 \quad \text { and }\left.\quad \frac{\partial q}{\partial r}\right|_{r=1}=\operatorname{Bi}\left[c(x, t)-L\left(q_{R}\right)\right]
$$

where $q_{R}(\boldsymbol{x}, t)=q(1, t ; \boldsymbol{x})=q_{R}^{\prime}\left(\boldsymbol{x}^{\prime}, t^{\prime}\right) / q_{\mathrm{ref}} ;$ and $q_{R}^{\prime}=$ value of the adsorbed mass on external particle surfaces. Eqs. (3) and (4) are a boundary value problem in $r$ that has to be solved for each $\boldsymbol{x}$.

\section{Numerical Solver}

The system of partial differential Eqs. (2)-(4) defines the HSDM model. Several approaches have been proposed to solve the model numerically, most of which are finite difference schemes for the two equations (Weber and Crittenden 1975; Oimstead and Weber 1990; Smith 1991; Sun et al. 1996; Başağaoğlu et al. 2000; Sperlich et al. 2008; Sonetaka et al. 2009a, b) or mixed methods: finite difference schemes for the transport-reaction equation and orthogonal collocation schemes for the intraparticle diffusion equation (Baup et al. 2000) or Laplace transformation and orthogonal collocation schemes (Roy et al. 1993). Analytical approximations have also been presented for simplified problems (Hand et al. 1984; Sperlich et al. 2008).

A novel approach is proposed in this paper: the intraparticle diffusion equation is reduced to a system of two ODEs, the 
transport-reaction equation is discretized by using a discontinuous Galerkin (DG) method, and the overall system evolution is integrated with a time-marching scheme. Thanks to the DG method, it is possible to transport smooth but also nonsmooth variations of the solution by introducing an element-by-element variable diffusion, in contrast with classical stabilized finite differences and finite elements schemes that tend to spread the solution with higher gradients along the whole domain, obtaining hence, smooth sharp profiles. As shown in next section, the proposal approximate adequately model solutions for a wide range of dimensionless numbers.

To simplify Eqs. (3) and (4) into an ODE system, a Galerkin spatial discretization is applied. Intraparticle diffusion $q(r, t ; \boldsymbol{x})$ is approximated by a polynomial of degree $m \geq 2$ in $r$

$$
q(r, t ; \boldsymbol{x})=q_{P}(\boldsymbol{x}, t) \frac{3+m}{m}\left(1-r^{m}\right)+q_{R}(\boldsymbol{x}, t) \frac{1}{m}\left[(3+m) r^{m}-3\right]
$$

The unknown of Eqs. (3) and (4) changes from $q(r, t ; \boldsymbol{x})$ to the couple of variables $q_{P}(\boldsymbol{x}, t)$ and $q_{R}(x, t)$. After some arithmetical operations, the following system of two coupled ODEs is obtained:

$$
\begin{aligned}
D_{g} \frac{\partial q_{P}}{\partial t}= & 3 \operatorname{St}\left[c-L\left(q_{R}\right)\right] \\
D_{g} \frac{\partial q_{R}}{\partial t}= & (2 m+6) \operatorname{St}\left[c-L\left(q_{R}\right)\right] \\
& +\frac{3 m^{3}+15 m^{2}+36 m+27}{2 m+1} \operatorname{Ed}\left(q_{P}-q_{R}\right)
\end{aligned}
$$

Eq. (6) is explicitly coupled with transport-reaction Eq. (2) through $c(\boldsymbol{x}, t)$ and $q_{P}(\boldsymbol{x}, t)$. The overall system, Eqs. (2) and (6), is linear for linear isotherms, $n=1$.

A polynomial of degree $m$ was used to simplify the intraparticle diffusion equation in reducing the microscale model by Galerkin approximation. Second-order approximation, $m=2$, works fine in many situations, but some authors have analyzed different contexts and models and concluded by proposing degrees higher than two (Li and Yang 1999; Sircar and Hufton 2000;
Başağaoğlu et al. 2000; Gadre et al, 2005). To obtain a more accurate approximation, a variable value of $m$, function of $\xi=\log (\mathrm{Ed})=\log (\mathrm{St} / \mathrm{Bi})$, is proposed in this paper. The value of $m$ is fixed equal to two for $\xi \geq 0$ (or equivalently $\mathrm{St} \geq \mathrm{Bi}$ ), and the following expression is proposed for $\xi \leq 0(\mathrm{St} \leq \mathrm{Bi})$ :

$$
m(\xi)=0.389 \cdot \xi^{4}-0.336 \cdot \xi^{3}+1.275 \cdot \xi^{2}+2
$$

Eq. (7) was adjusted by comparing the results of the reduced model, Eq. (6), with those of the full intraparticle model, Eqs. (3) and (4). A continuous feed test with a dimensionless final time equal to $D_{g}$ was used for comparison. The test is defined imposing $c(\boldsymbol{x}, t)=1$ in Eq. (4) or in Eq. (6). Both problems were solved numerically with very high precision. The evolution of mean adsorbed mass, $q_{P}$, was used to measure the goodness of the approximation. The best $m$ values were chosen for each set of dimensionless numbers. The following values were used to adjust Eq. (7): $m(0)=2$, $m(-1)=3, m(-2)=16, m(-3)=54$, and $d m /\left.d \xi\right|_{\xi=0}=0$ to present a smooth transition at $\xi=0$. The proposal is independent of $\mathrm{Bi}$; thus, it should be applied for all values of $\mathrm{Bi}$. However, tests showed that the problem can be adequately simulated with $m=2$ for $\mathrm{Bi} \leq 10$.

The spatial discretization of the transport-reaction equation is performed by using the Local Discontinuous Galerkin (LDG) scheme (Cockburn and Shu 1998; Sherwin et al. 2006). It is well known that DG methods are well-suited for hyperbolic and convection-dominated problems, thanks to their natural stabilization caused by numerical fluxes. This fact also makes them a good approach for the treatment of sharp profiles. Morevover, the discontinuous nature of the method allows to add local stabilization and to change the interpolation within each element hence, adapting the approximation to the solution profile along the spatial and time discretization. In HSDM simulations, nonlinear isotherms and a wide range of values of dimensionless numbers lead the profiles of the unknowns, $c, q_{P}$, and $q_{R}$ to contain shocks and sharp profiles.

The simulation of shocks and the advection of sharp fronts constitute a key problem in numerical methods (Harten 1983; Shu 1988; Dawson and Aizinger 2005) because they lead to spurious oscillations and unstable solutions unless a specific mechanism is
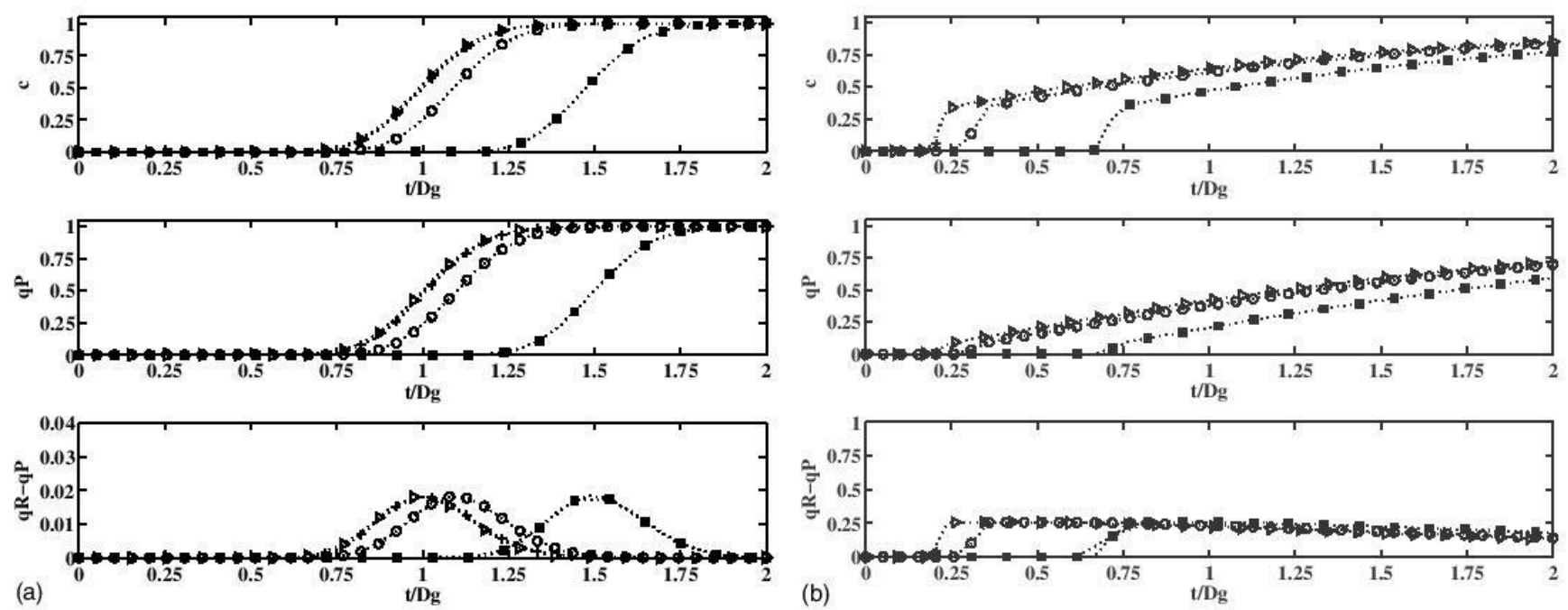

- $D g=2$ a $D g=10 \cdots+D g=100 \cdots D g=1000$

Fig. 1. Breakthrough curves of $c, q_{P}$, and $q_{R}-q_{P}$ for $D_{g}$ from 2 to $10^{3}$ and $n=1:$ (a) $\mathrm{Bi}=10$ and $\mathrm{St}=10^{2} ;$ (b) $\mathrm{Bi}=10^{3}$ and $\mathrm{St}=10$ 
used. In this work, the artificial diffusion method for DG approximations is used (Casoni et al. 2013). Although DG schemes introduce a natural dissipative mechanism through the jump terms (Persson and Peraire 2006; Huerta et al. 2012), an improved stabilization technique is needed when strong shocks are present to avoid Gibbs oscillations. The introduction of an artificial diffusion method combined with a sensor based on the smoothness of the solution makes it possible to introduce a minimum, but enough, amount of diffusion to avoid spurious oscillations only in the regions where sharp gradients are detected. This is in contrast with standard finite differences and finite-element numerical methods, in which either the dissipative effect takes place along the whole domain and hence, the sharp profile is lost, either add a paramenterdependent diffusion that is not robust and must be tuned depending on the problem. For more details see (Donea and Huerta 2003).

All the simulations in this paper were performed with a mesh of 100 linear elements. The following sections show that this is enough for simple plug-flow simulations. Higher degrees can be easily applied (Casoni et al. 2013). Time integration of the overall system is done by using the forward Euler method. Higher-order time-marching schemes are also possible.
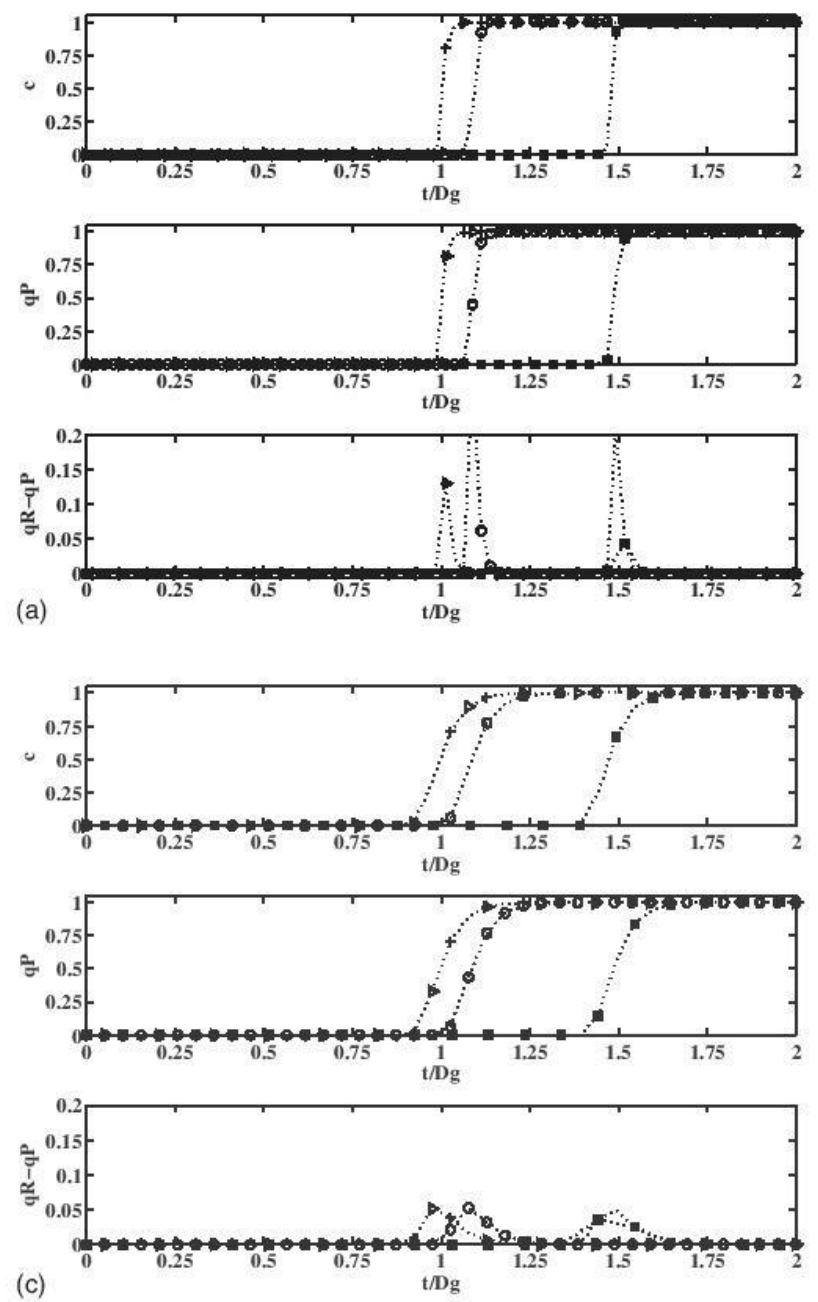

(b)
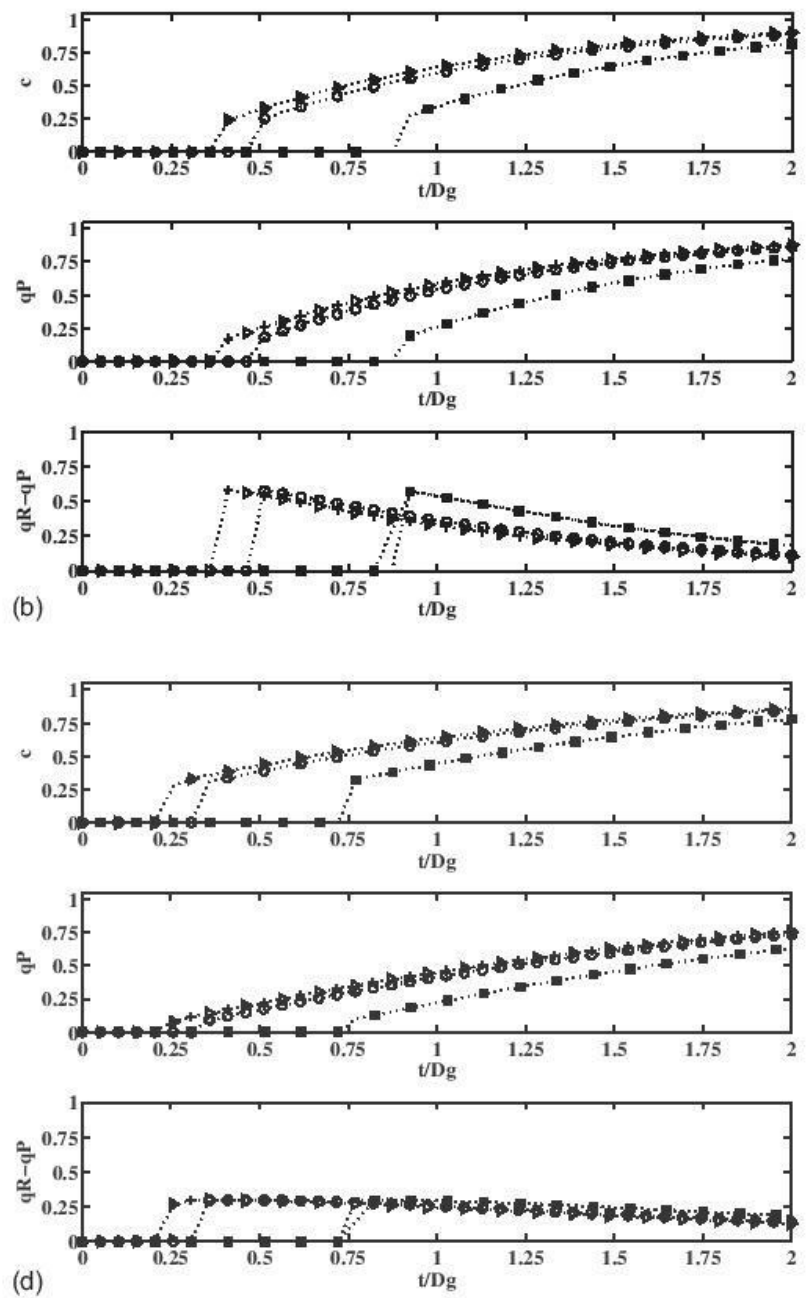

(d)

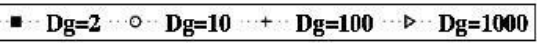

Fig. 2. Breakthrough curves of $c, q_{P}$, and $q_{R}-q_{P}$ for $D_{g}$ from 2 to $10^{3}$ : (a) $\mathrm{Bi}=10, \mathrm{St}=10^{2}$ and $n=0.2 ;$ (b) $\mathrm{Bi}=10^{3}, \mathrm{St}=10$ and $n=0.2$; (c) $\mathrm{Bi}=10, \mathrm{St}=10^{2}$ and $n=0.8 ;(\mathrm{d}) \mathrm{Bi}=10^{3}, \mathrm{St}=10$ and $n=0.8$ 
The BTCs of $c$, the standard concentration outlet, but also the evolution of $q_{P}$ and the difference $q_{R}-q_{P}$ at the end of the domain, are shown. The difference $q_{R}-q_{P}$ is a measure of the uniformity distribution of the adsorbed mass inside the particles.

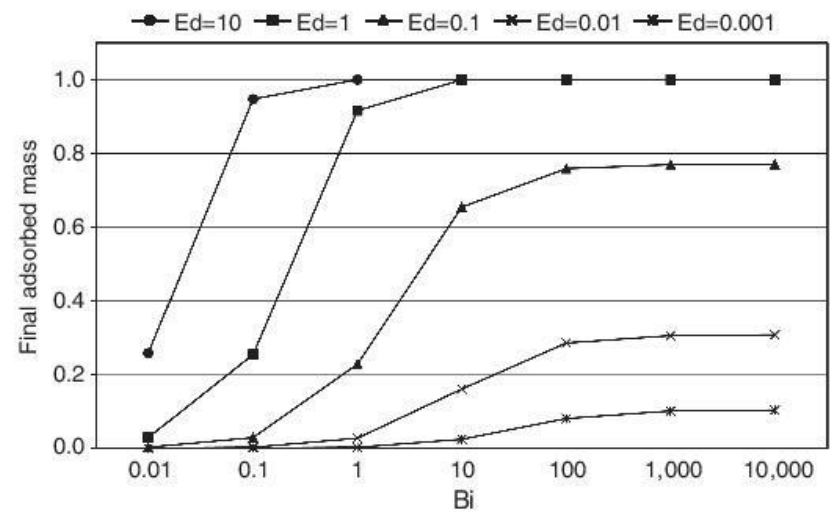

Fig. 3. Continous feed test, adsorbed mass inside particles at $t_{\text {fin }}=D_{g}$ for $n=1$ and Ed from $10^{-3}$ to $10^{1}$
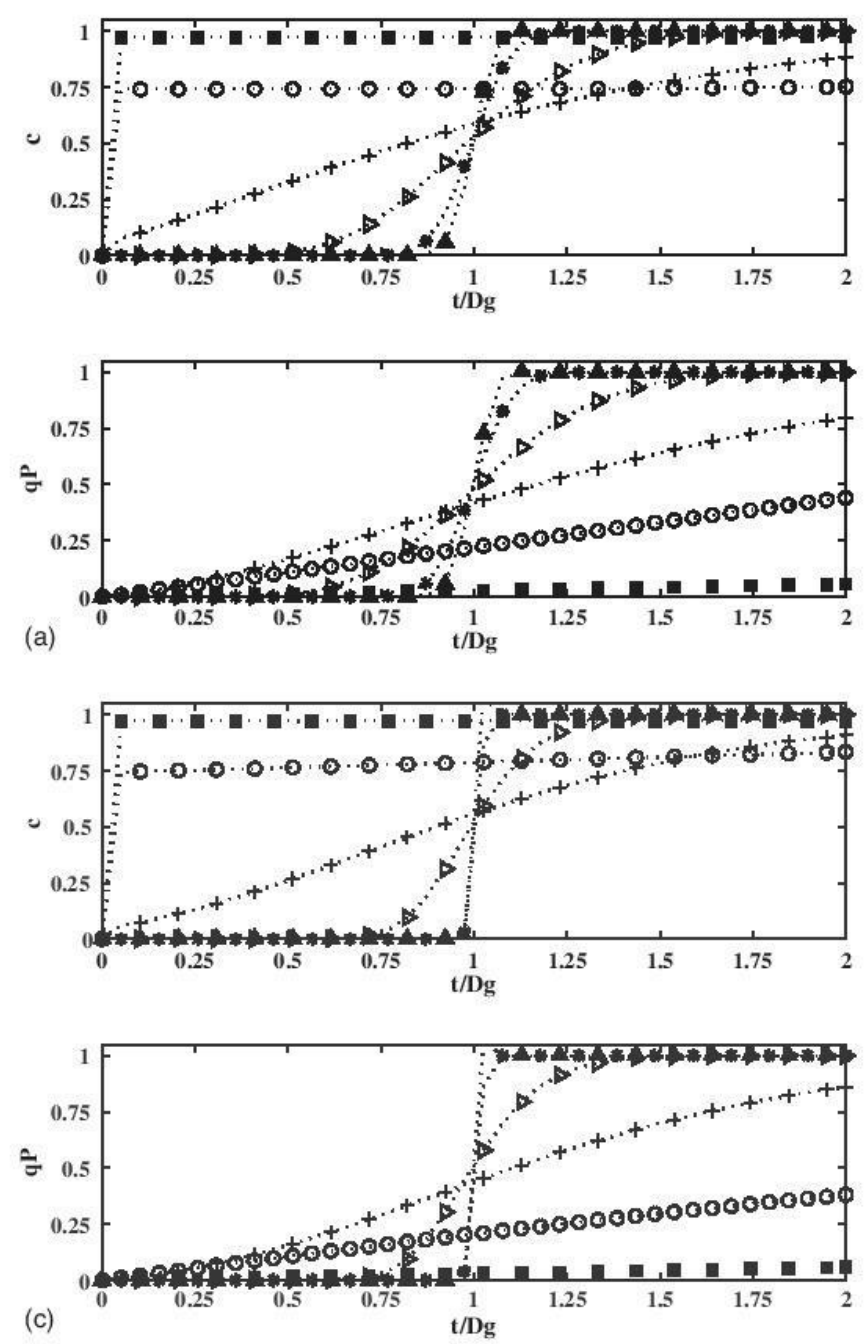

In left-hand cases depicted in Figs. 1 and $2, \mathrm{Bi}=10$ and $\mathrm{St}=10^{2}$, adsorption is faster than convection. $\mathrm{St}=10^{2}$ indicates that film mass transfer process is two orders of magnitude faster than convection, and $\mathrm{Bi}=10$ indicates that internal diffusion is just one order of magnitude slower than film mass transfer (and therefore, one order of magnitude faster than convection, $\mathrm{Ed}=$ $\mathrm{St} / \mathrm{Bi}=10)$. In this situation, the BTCs of $c$ and $q_{P}$ are indistinguishable and consistently with this, the difference $q_{R}-q_{P}$ is close to zero. This difference is larger for the nonlinear case, especially for lower values of $n$. However, the general behavior of the model in $c$ and $q_{P}$ is similar.

Instead, in right-hand cases shown in Figs. 1 and 2, film mass transfer process is one order of magnitude faster than convection, $\mathrm{St}=10$, but $\mathrm{Ed}=\mathrm{St} / \mathrm{Bi}=10^{-2}$ indicates that internal surface diffusion is two orders of magnitude slower than convection $\left(\mathrm{Bi}=10^{3}\right.$ indicates that it is three orders of magnitude slower than film mass transfer). Fast film mass transfer and slow internal diffusion processes are identified in right-hand side of Figs. 1 and 2, through a smoother BTC of $q_{P}$ than of $c$, a step at an early stage of the BTC of $c$, and larger values of $q_{R}-q_{P}$ than previous fast adsortion cases. In this situation, the two adsorption processes occur at different time-scales, separated by convection process, and BTCs
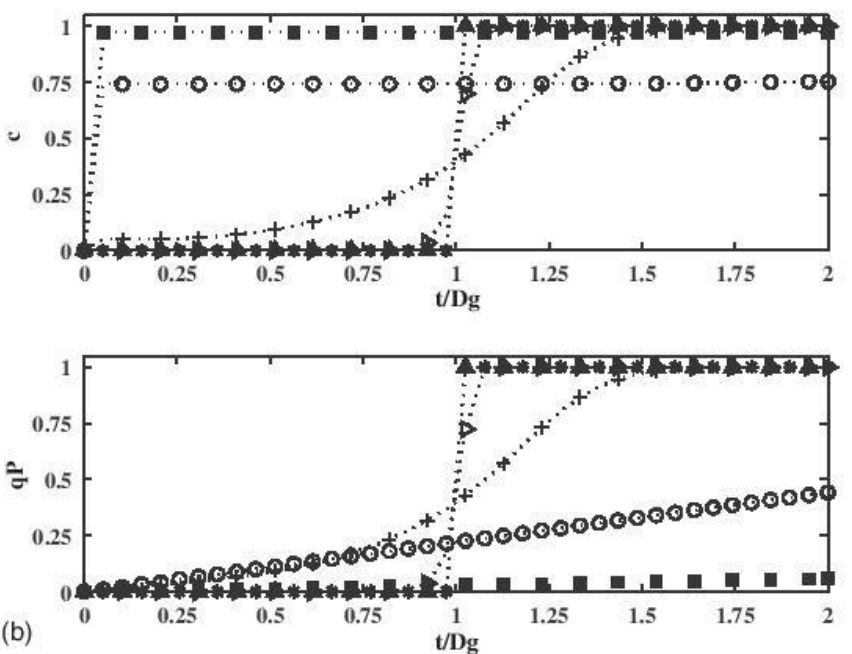

Fig. 4. Breakthrough curves of $c$ and $q_{P}$ for $\mathrm{Bi}=10^{-1}, n=1,0.2$ and 0.8 and $\mathrm{St}$ from $10^{-2}$ and $10^{3}$ : (a) $n=1$; (b) $n=0.2$; (c) $n=0.8$ 
reflect it. Large film mass transfer induces a fast growing of $q_{R}$, from zero to 1, but low values of Ed keep differences between $q_{P}$ and $q_{R}$ large because internal diffussion does not have influence at this time scale. Diferences $q_{P}-q_{R}$ reduce with time, asymptotically to zero.

Values of $D_{g}$ can be very large in highly adsorbent media, up to $10^{5}$. Because the final simulating time is proportional to $D_{g}$, simulation of highly adsorbent media can be expected to involve a large computational time. As shown in left-hand cases of Figs. 1 and 2, the solutions of fast adsorption processes are smooth waves traveling at a constant velocity equal to $1 /\left(1+D_{q}\right)$, which, for large values of $D_{g}$, is approximately $D_{g}^{-1}$. The BTCs of $c$ and $q_{P}$ for $D_{g}=10^{2}$ and $D_{g}=10^{3}$ are indistinguishable. In this study, unless explicitly indicated, $D_{g}=10^{3}$ is considered. The wavefront position computed with this value will present an negligible delay of up to $10^{-3}$ with respect to results computed with higher values of $D_{g}$.

Finally, notice that low values of $n$ have relevant influence on shape and smoothness of BTC, but they have not on the general conclusions of this subsection about model behavior and influence of $D_{g}$ on results. Nonlinearity does not change the overall dimensionless behavior of the model.
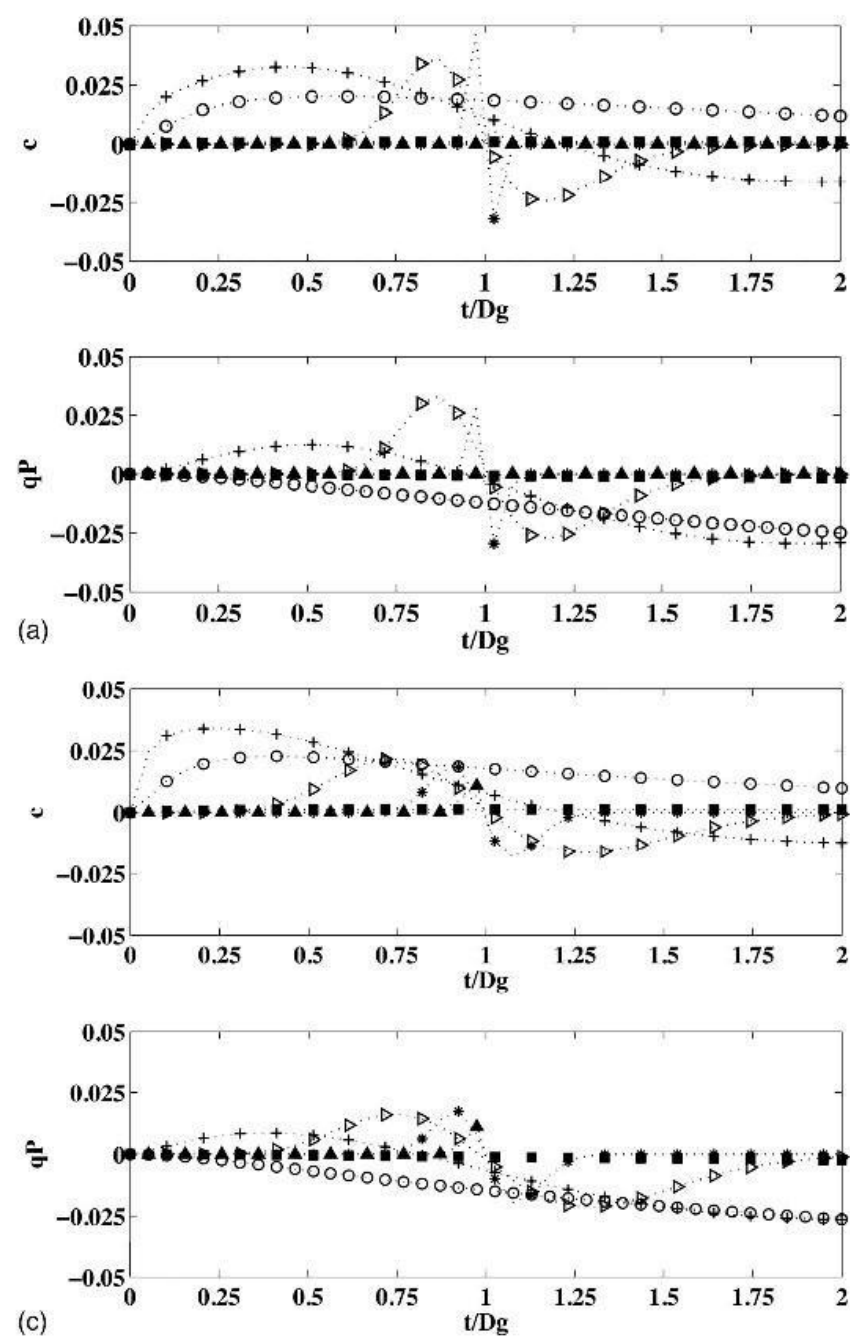

\section{Influence of Ed}

Before going further in the analysis of BTCs, in this subsection, the influence of Ed is assessed simulating continous feed conditions. The adsorbed mass inside particles at $t_{\mathrm{fin}}=D_{g}$ for the linear case, $n=1$, and different values of $\mathrm{Ed}$ and $\mathrm{Bi}$ are presented in Fig. 3. The final adsorbed mass is close to one in all cases with fast adsorption processess: $\mathrm{Bi} \leq 10^{0}$ (faster internal diffusion than film mass transfer) and $\mathrm{St}=\mathrm{EdBi} \geq 10^{\circ}$ (faster film mass transfer than fixed problem time-scale); and $\mathrm{Bi} \geq 10^{\circ}$ and $\mathrm{Ed} \geq 10^{\circ}$ (faster film mass transfer than internal diffusion and this than problem time-scale). The final adsorbed mass is close to zero in all cases with slow adsorption processess.

Keeping the Bi value fixed, the larger the value of Ed, the faster the adsorption and larger the portion of adsorption capacity used during the time scale given by $D_{g}$. In the opposite direction, lower values of Ed preclude slower adsorption, and in this case, just a reduced part of the adsorption capacity is used, see $\mathrm{Ed} \leq$ $10^{-2}$ in Fig. 3. These results are coherent with those obtained before; compare with results of Figs. 1 and 2. At the time-scale given by $D_{g}$, small internal surface diffusions limit adsorption significatively.
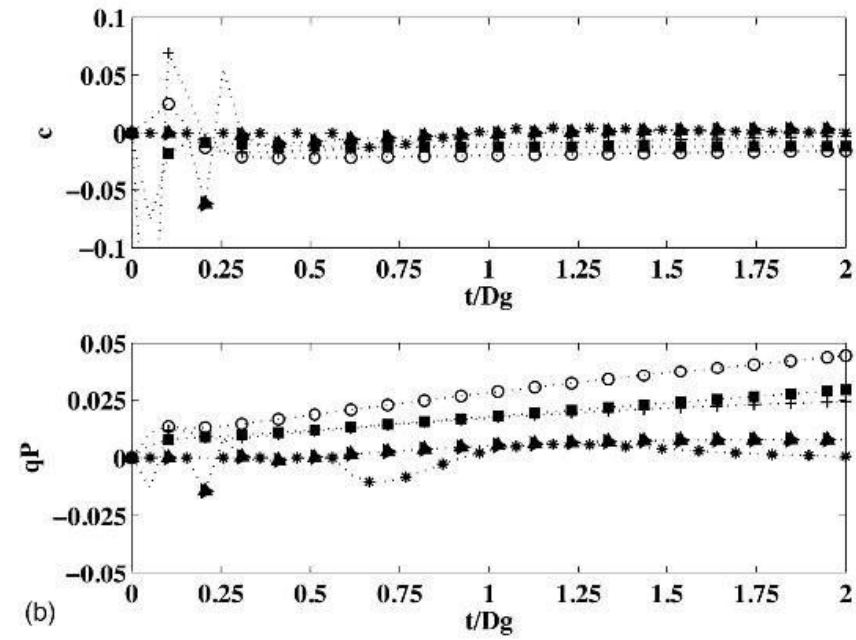

Fig. 5. Differences between breakthrough curves of $c$ and $q_{P}$ computed with $\mathrm{Bi}=10^{0}$ and $\mathrm{Bi}=10^{-1}$ for $n=1,0.2$, and 0.8 and common values of St from $10^{-2}$ and $10^{3}:$ (a) $n=1$; (b) $n=0.2$; (c) $n=0.8$ 


\section{Influence of Bi and St}

The BTCs obtained with small and large values of Bi are presented and analyzed for a wide range of and St and linear and nonlinear cases.

Fig. 4 shows BTCs for the cases $\mathrm{Bi}=10^{-1}$, St from $10^{-2}$ to $10^{3}$ $\left(\mathrm{Ed}=\mathrm{St} / \mathrm{Bi}\right.$ from $10^{-3}$ to $\left.10^{2}\right)$, and $n=1,0.8$ and 0.2 . Large values of St imply fast, even instantaneous, adsorption, and both $c$ and $q_{P}$ BTCs correspond to a front traveling at velocity $D_{g}^{-1}$, as results presented in the left-hand side of Figs. 1 and 2. Small values of St imply slow adsorption. The wavefront is smooth and just part of the medium is filled with adsorbent at this time scale. In the limit case of no adsorption, $\mathrm{St}=0$, a wavefront traveling at a velocity of one (much faster than $D_{g}^{-1}$ ) is obtained. A sharp front is found just at the beginning of the BTCs at a dimensionless time equal to one. This later case corresponds to a pure convection problem, and it is found in Fig. 4 for $\mathrm{St}=10^{-2}$.

Results for linear and nonlinear cases present similar shapes of the BTCs and similar transitions between the limit behaviors (instantaneous adsorption and no adsorption). Main differences are the sharpness of the front, which is accentuated for the nonlinear cases (low values of $n$ ), and the value of St that can be considered instantaneous adsorption. This value of St decreases with $n$ : St $=$ $10^{2}$ for $n=1$ and 0.8 and $10^{1}$ for $n=0.2$. It reduces inversely to nonlinearity. Transition between limit behaviors in St is more abrupt for nonlinear problems than for linear ones.

Similar BTCs are found with $\mathrm{Bi}=10^{0}$. Fig. 5 shows the differences between the results obtained with $\mathrm{Bi}=10^{\circ}$ and those obtained with $\mathrm{Bi}=10^{-1}$ (depicted in Fig. 4) for the same values of St and $n$. Error is less than $5 \%$ for all cases and during all time intervals, exceeding the limit of $2 \%$ of error only for specific values of St and punctual instants. Differences between the results obtained with smaller pairs of $\mathrm{Bi}$ (i.e., $10^{-1}$ with respect to $10^{-2}$ ) are also reduced. Therefore, a limit behavior is found for $\mathrm{Bi} \leq 10^{\circ}$, that is, when film mass transfer dominates the problem. In this limit case, BTCs depend only on St, which relates film mass transfer and convection velocities. It ranges from instantaneous adsorption to no adsorption (pure convection).

On the other hand, large values of $\mathrm{Bi}$ correspond to film mass transfer process faster than internal diffusion, and hence, internal surface diffusion dominates the adsorption behavior of the model. Fig. 6 shows the results for $\mathrm{Bi}=10^{3} ; \mathrm{Ed}=\mathrm{St} / \mathrm{Bi}$ from $10^{-3}$ to $10^{2}$; and $n=1,0.8$, and 0.2 . Although the same limit behaviors
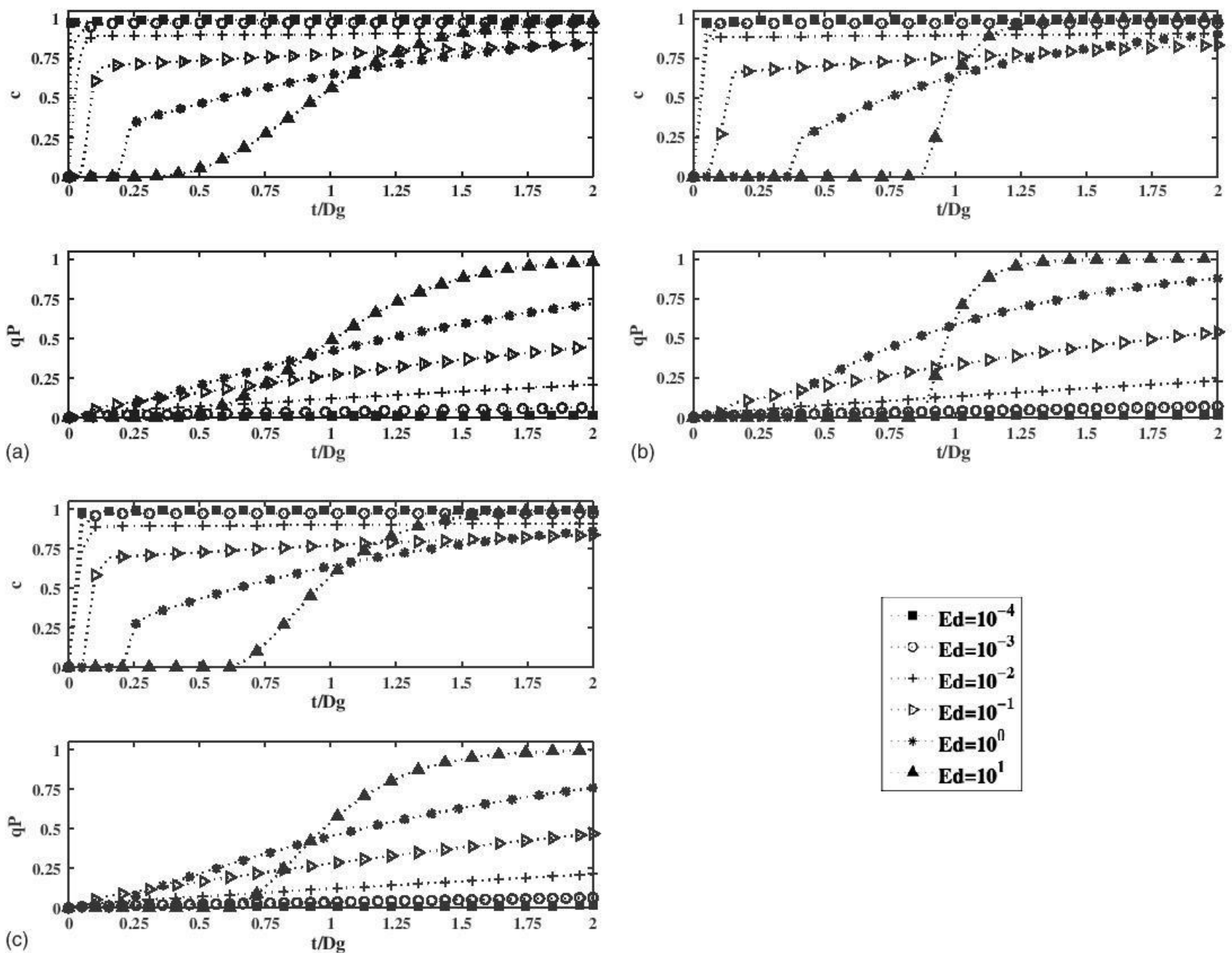

Fig. 6. Breakthrough curves of $c$ and $q_{P}$ for $\mathrm{Bi}=10^{3} ; n=1,0.2$, and 0.8 ; and $\mathrm{Ed}$ from $10^{-4}$ to $10^{1}$ : (a) $n=1$; (b) $n=0.2$; (c) $n=0.8$ 
as in Fig. 4 are identified, intermediate solutions show different behavior in the two Figs. 4 and 6. A step is identified at an early stage of the BTC of $c$, concentration outlet, for $\mathrm{Ed} \leq 10^{\circ}$. Position and sharpness of this step depend on $n$ for $\mathrm{Ed} \in\left[10^{-1}, 10^{0}\right]$. Values of Ed $\leq 10^{-2}$ present a significantly reduced adsorption at this time scale; see final values of $q_{P}$ in Fig. 6. These results are coherent with those of a previous subsection (Fig. 3). Adsorbed mass $q_{P}$ presents a smoother BTC than $c$, and constant values close to zero are found for small values of Ed.

Fig. 7 presents results obtained with $\mathrm{Bi}=10^{3}$ minus those obtained with $\mathrm{Bi}=10^{2}$ for the same values of $\mathrm{Ed}$ and $n$. The overall differences are close to zero throughout all BTCs, except in the front position, where slightly noticeable differences can be seen (punctual errors even larger than $10 \%$ are detected when comparing BTCs). They are caused by small differences in the position of the initial step. The same results are obtained when comparing results with larger values of $\mathrm{Bi}$. Thus, a limit behavior is found for $\mathrm{Bi} \geq 10^{2}$, that is, when internal surface diffusion dominates the problem. In this limit case, BTCs depend only on Ed, which relates internal surface diffusion and convection velocities. It ranges from instantaneous adsorption to no adsorption (pure convection) analogously to the other limit obtained before, $\mathrm{Bi} \leq 10^{\circ}$, in which solution depends on St.
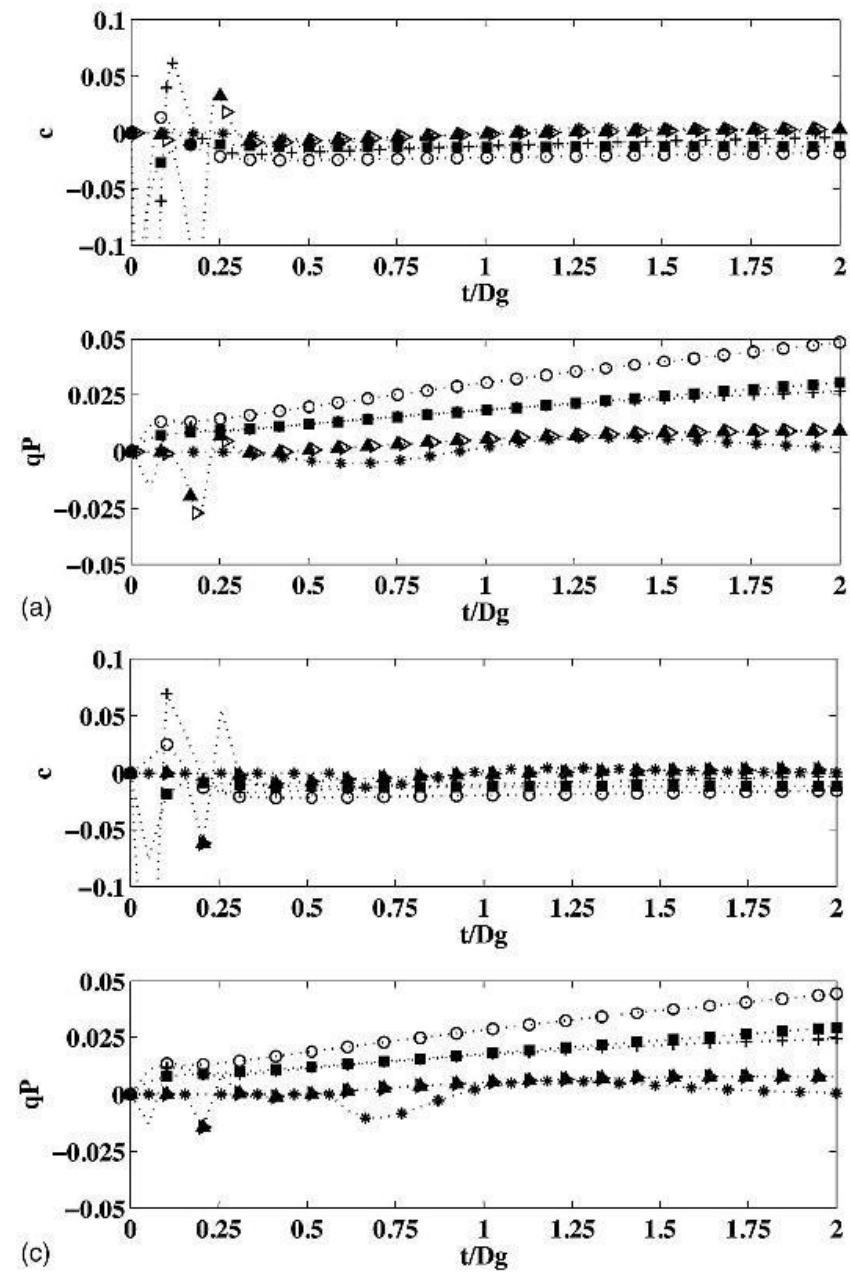

\section{GFH Characterization with HSDM}

Experimental adsorption results for different adsorbates in GFH were reproduced with HSDM in Sperlich et al. (2008). Characteristic dimensionless numbers of these cases are: large values of $D_{g}$, from $10^{3}$ to $10^{5}$; nonlinear isotherms, $n<1$; values of $\mathrm{Bi}$ from $10^{1}$ to $10^{3}$; and $\mathrm{Ed}=\mathrm{St} / \mathrm{Bi}$ from $10^{-2}$ to $10^{0}$. Thus, the HSDM behavior is close to the limit detected for high $\mathrm{Bi}$ and relatively low values of Ed. The BTCs with shapes similar to those shown in Fig. 6 are expected.

Fig. 8 shows the BTCs of four adsorbates (salicylic acid, DOC, phosphate, and arsenate) computed with the HSDM formulation presented in this paper, together with the experimental data and the simulation results presented by Sperlich et al. (2008). Table 1 summarizes the parameters that define these examples. Computations have been done with the parameters of Table 1, with the exception of $D_{g}$, which is limited to $10^{3}$ in all cases because the dimensionless problem is independent of $D_{g}$ for large $D_{g}$ values.

In three of the four cases, similar results were obtained with the HSDM formulation presented in this paper and with that used by Sperlich et al. (2008). However, in the case of arsenate, the differences were significant. This is probably caused by the overdiffusive character of the numerical solver used in previous
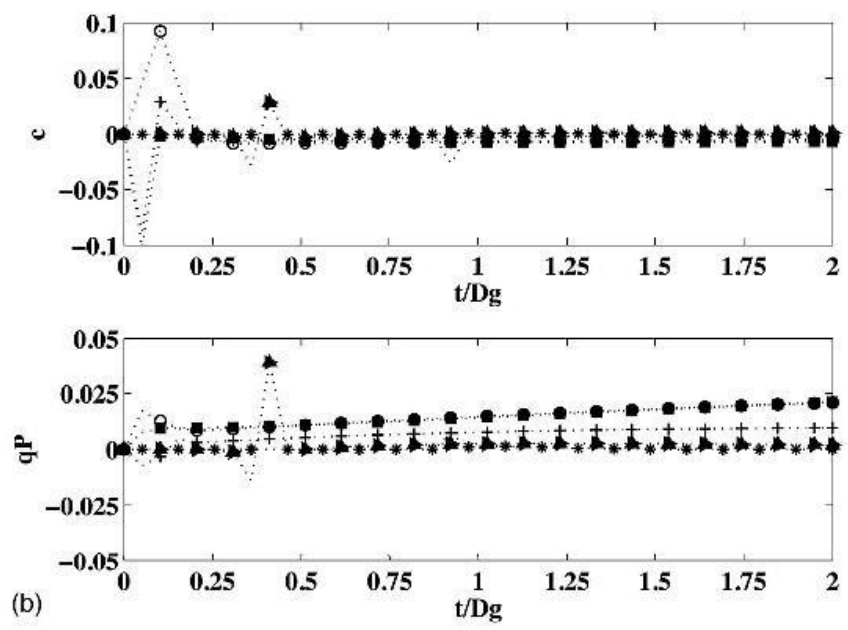

Fig. 7. Differences between breakthrough curves of $c$ and $q_{P}$ computed with $\mathrm{Bi}=10^{3}$ and $\mathrm{Bi}=10^{2}$ for $n=1,0.2$, and 0.8 and common values of $\mathrm{Ed}$ from $10^{-4}$ to $10^{1}$ : (a) $n=1$; (b) $n=0.2$; (c) $n=0.8$ 

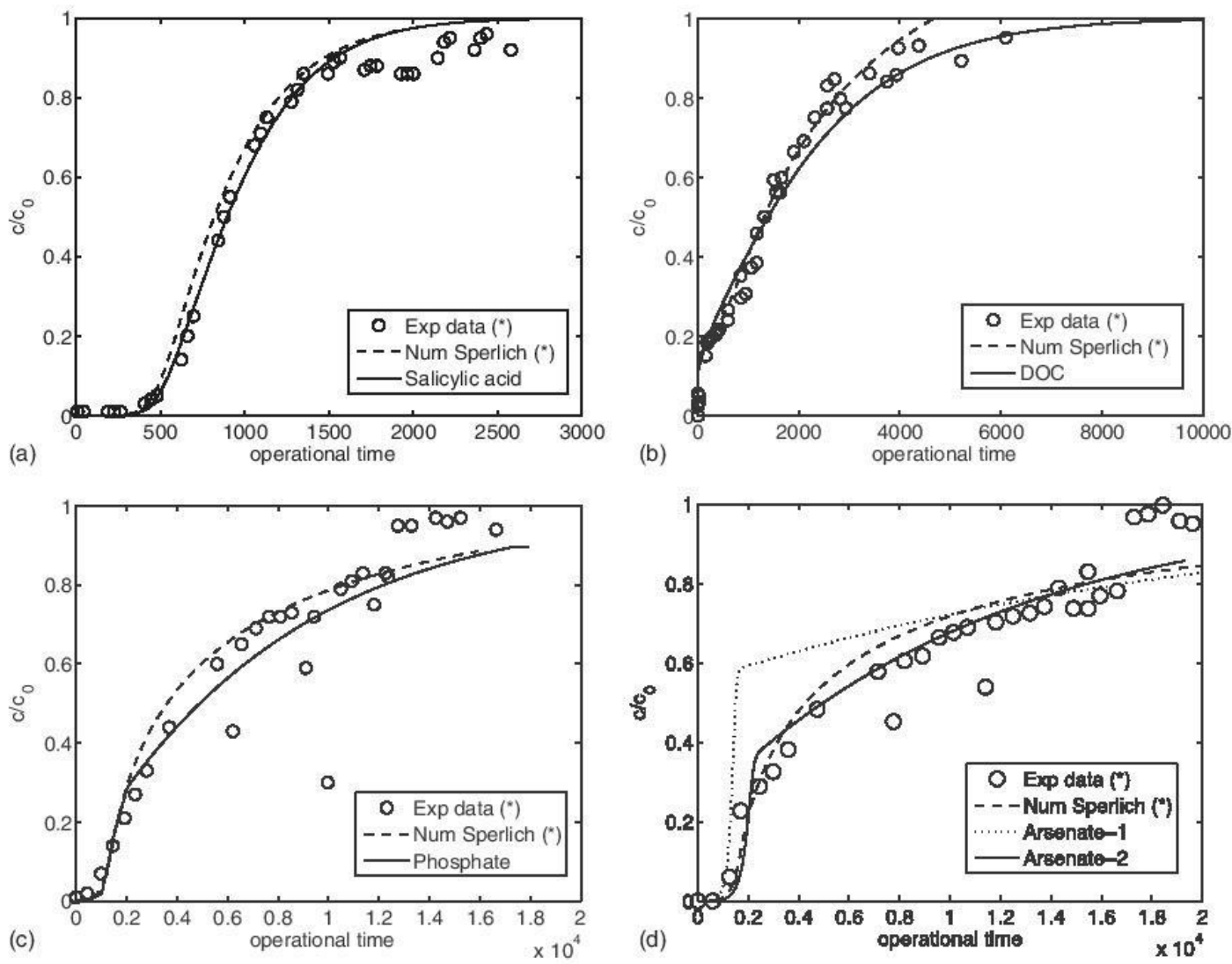

Fig. 8. Experimental data and HSDM simulations for (a) salicylic acid; (b) DOC; (c) phosphate; (d) arsenate; results presented in Sperlich et al. (2008) are indicated with $(*)$; values from Table 1 are used in simulations

Table 1. GFH Data for HSDM Simulations

\begin{tabular}{lcrcrcc}
\hline Example & \multicolumn{1}{c}{$B_{i}$} & \multicolumn{1}{c}{$S_{t}$} & Ed & \multicolumn{1}{c}{$D_{g}$} & $n$ & $R$ \\
\hline Salicylic acid & 16.3 & 6.9 & 0.4233 & 4,155 & 0.52 & 0.39 \\
DOC & 23.3 & 3.9 & 0.1673 & 8,140 & 0.62 & 0.39 \\
Phosphate & 102.1 & 10.0 & 0.0979 & 20,323 & 0.19 & 0.45 \\
Arsenate-1 & 182.5 & 9.7 & 0.0532 & 24,617 & 0.19 & 0.45 \\
Arsenate-2 & 91.25 & 4.5 & 0.0532 & 1,000 & 0.19 & 0.45 \\
\hline
\end{tabular}

Note: Data from Sperlich et al. (2008), except Arsenate-2, from this study.

computations, which influences results when sharp profiles are developed. Simulation with $\mathrm{Bi}$ (and $\mathrm{St}$ ) halved with respect to the original values is presented for arsenate (named Arsenate- 2 in Table 1). Thus, the value of Ed is kept in common between the two arsenate simulations. A later one fits much better with the experimental data than the first one, computed with the original values of Sperlich et al. (2008). The sharp variation in BTCs slope, also present in exprimental data, reflects the different time-scale of the two processes that define adsorption in HSDM, film mass transfer, and internal surface diffusion.

\section{Conclusions}

The HSDM has been fully characterized in this study, including a detailed description of its limit behaviors. In this paper, previous limits in $\mathrm{Bi}$ are confirmed but complemented by the influence of St and Ed.
An extensive analysis of breakthrough curves for linear and nonlinear isotherms has been presented. Interesting results have been found. The BTCs depend on St for $\mathrm{Bi} \leq 10^{\circ}$ but on $\mathrm{Ed}=$ $\mathrm{St} / \mathrm{Bi}$ for $\mathrm{Bi} \geq 10^{2}$. In both cases, BTCs are independent of Bi values; BTCs depend only on St or Ed, respectively. Adsorption is immediate for large values of St (or correspondingly of Ed), and on the contrary, a pure convection problem is found (that is, no adsorption) for small values of both St and Ed. In between, both $\mathrm{Bi}$ and St (or Ed) have an influence on BTCs. In the linear case, for low values of $\mathrm{Bi}$, BTCs depend on St within the range from $10^{-2}$ to $10^{2}$; and for large $\mathrm{Bi}$, the results depend on $\mathrm{Ed}$ within the range from $10^{-2}$ to $10^{2}$. The same conclusions are found with nonlinear cases but with slightly narrower limits.

In BTCs for a large $\mathrm{Bi}$, a step is found at an early stage of the concentration outlet for relatively low values of $\mathrm{Ed}, 10^{-2}$ to $10^{-1}$, because of different time-scales of film mass transfer and intraparticle surface diffusion processes. When smaller values of Ed are used, no adsorption takes place, and this step collapses with convective transport at the beginning of the BTC.

A novel numerical solver has also been presented. The intraparticle diffusion equation has been simplified with a Galerkin approximation in a variable order polinomial. The adaptive degree of interpolation, defined as a function of the dimensionless numbers, has been used to capture the main local-level characteristics at the BTC time scale. A discontinuous Galerkin method for space discretization of transport-reaction equation has also been proposed. This scheme is able to capture sharp gradients, advecting the shock at the correct speed and without adding dissipation significatively. This is crucial for nonlinear isotherms and cases with 
large $\mathrm{Bi}$ and small $\mathrm{Ed}$ because the solution contain shocks and sharp profiles, and hence, a highly accurate numerical solver is required to correctly simulate these cases.

The proposed HSDM formulation has been shown to be capable of characterizing a wide range of adsorption processes. The BTCs for several adsorbates onto GFH have been predicted. The simulations with HSDM reproduce the experimental data and also agree with previous results published in the literature (Sperlich et al. 2008). The same characteristic dimensionless numbers have been used, except for the highest Biot numbers, where the dimensionless numbers Bi and St had to be halved to better fit the data, but the Ed value was kept constant. Hence, the GFH simulations agree with the expected results, confirming the full characterization of the model here proposed.

\section{References}

Badruzzaman, M., Westerhoff, P., and Knappe, D. (2004). "Intraparticle diffusion and adsorption of arsenate onto granular ferric hydroxide (GFH)." Water Res., 38(18), 4002-4012.

Başağaoğlu, H., Ginn, T., McCoy, J., and Mariño, M. (2000). "Linear driv ing force approximation to a radial diffusive model." AIChE J., 46(10), 2097-2105.

Baup, S., Jaffre, C., Wolbert, D., and Laplanche, A. (2000). "Adsorption of pesticides onto granular activated carbon: Determination of surface diffusivities using simple batch experiments." Adsorption, 6(3), 219-228.

Brattebo, H., and Odegaard, H. (1986). "Phosphorus removal by granular activated alumina." Water Res., 20(8), 977-986.

Brusseau, M., and Gillham, P. R. R. W. (1989). "Sorption nonideality during organic contaminant transport in porous media." Crit. Rev. Env. Sci. Technol., 19(1), 33-99.

Casoni, E., Peraire, J., and Huerta, A. (2013). "One-dimensional shockcapturing for high-order discontinuous Galerkin methods." Int. J. Numer. Methods Fluids, 71(6), 737-755.

Chang, S., Waite, T., Ong, P., Schfer, A., and Fane, A. (2004). "Assessment of trace estrogenic contaminants removal by coagulant addition, powdered activated carbon adsorption and powdered activated carbon microfiltration processes." J. Environ. Eng., 130(7), 736-742.

Cockburn, B., and Shu, C.-W. (1998). "The local discontinuous Galerkin method for time-dependent convection-diffusion systems." SIAM J. Numer. Anal., 35(6), 2440-2463.

Crittenden, J., Berrigan, J., Hand, D., and Lykins, B. (1987). "Design of rapid fixed-bed adsorption tests for nonconstant diffusivities." J. Environ. Eng., 113(2), 243-259.

Dawson, C., and Aizinger, V. (2005). "A discontinuous Galerkin method for three-dimensional shallow water equations." J. Sci. Comput., 22-23(1), 245-267.

Donea, J., and Huerta, A. (2003). Finite element methods for flow prob lems, Wiley, Chichester, UK.

Flora, J., Vidic, R., Liu, W., and Thumau, R. (1998). "Modeling powdered activated carbon injection for the uptake of elemental mercury vapors." J. Air Waste Manage. Assoc., 48(11), 1051-1059.

Gadre, S., Ebner, A., and Ritter, J. (2005). "Further validation of the quartic concentration profile approximation for describing intraparticle transport in cyclic adsorption processes." Adsorption, 11(3-4), 295-314.

Genz, A., Baumgarten, B., Goemitz, M., and Jekel, M. (2008). "NOM removal by adsorption onto granular ferric hydroxide: Equilibrium, kinetics, filter and regeneration studies." Water Res., 42(1-2), 238-248.

Hand, D., Crittenden, J., and Thacker, W. (1983). "User-oriented batch reactor solutions to the Homogeneous Surface Diffusion Model." J. Environ. Eng., 109(1), 82-101.

Hand, D., Crittenden, J., and Thacker, W. (1984). "Simplified models for design of fixed-bed adsorption systems.” J. Environ. Eng., 110(2), $440-456$.

Harten, A. (1983). "High resolution schemes for hyperbolic conservation laws." J. Computational Phys., 49(3), 357-393.
Huerta, A., Casoni, E., and Peraire, J. (2012). "A simple shock-capturing technique for high-order discontinuous Galerkin methods." Int. J. Numer. Methods Fluids, 69(10), 1614-1632.

Lee, M., Crittenden, J., Snoeyink, V., and Ari, M. (1983). "Design of carbon beds to remove humic substances." J. Environ. Eng., 109(3), 631-645.

Li, Z., and Yang, R. (1999). "Concentration profile for linear driving force model for diffusion in a particle." AIChE J., 45(1), 196-200.

Oimstead, K., and Weber, W. (1990). "Statistical analysis of mass-transfer parameters for sorption processes and models." Environ. Sci. Technol., 24(11), 1693-1700

Persson, P.-O., and Peraire, J. (2006). "Sub-cell shock capturing for discontinuous Galerkin methods." Collection of Technical Papers44th AIAA Aerospace Sciences Meeting, Vol. 2, AIAA, Reston, VA, 1408-1420.

Rahman, M., Amiri, F., and Worch, E. (2003). "Application of the mass transfer model for describing nonequilibrium transport of HOCs through natural geosorbents." Water Res., 37(19), 4673-4684.

Roy, D., Wang, G., and Adrian, D. (1993). "A simplified solution technique for carbon adsorption model." Water Res., 27(6), 1033-1040.

Sherwin, S. J., Kirby, R. M., Peiró, J., Taylor, R. L., and Zienkiewicz, O. C. (2006). "On 2D elliptic discontinuous Galerkin methods." Int. J. Numer. Methods Eng., 65(5), 752-784.

Shu, C.-W. (1988). "Total-variation-diminishing time discretizations." SIAM J. Sci. Stat. Comput., 9(6), 1073-1084.

Sircar, S., and Hufton, J. (2000). "Intraparticle adsorbate concentration profile for linear driving force model." AIChE J., 46(3), 659-660.

Smith, E. (1991). "Modified solution of homogeneous surface diffusion model for adsorption." J. Environ. Eng., 117(3), 320-338.

Smith, E. (1996). "Uptake of heavy metals in batch systems by a recycled iron-bearing material." Water Res., 30(10), 2424-2434.

Smith, E. (1997). "Wave front analysis for design of fixed-bed adsorbers." Chem. Eng. Commun., 159(1), 17-37.

Sonetaka, N., Fan, H.-J., Kobayashi, S., Su, Y.-C., and Furuya, E. (2009a). "Characterization of adsorption uptake curves for both intraparticle diffusion and liquid film mass transfer controlling systems." J. Hazard. Mater., 165(1-3), 232-239.

Sonetaka, N., Fan, H.-J., Kobayashi, S., Su, Y.-C., and Furuya, E. (2009b). "Simultaneous determination of intraparticle diffusivity and liquid film mass transfer coefficient from a single-component adsorption uptake curve." J. Hazard. Mater., 164(2-3), 1447-1451.

Sperlich, A., et al. (2008). "Predicting anion breakthrough in granular ferric hydroxide (GFH) adsorption filters." Water Res., 42(8-9), 2073-2082.

Sperlich, A., Werner, A., Genz, A., Amy, G., Worch, E., and Jekel, M. (2005). "Breakthrough behavior of granular ferric hydroxide (GFH) fixed-bed adsorption filters: Modeling and experimental approaches." Water Res., 39(6), 1190-1198.

Sun, L., Queré, P. L., and Levan, M. (1996). "Numerical simulation of diffusion-limited PSA process models by finite difference methods." Chem. Eng. Sci., 51(24), 5341-5352.

Traegner, U., and Suidan, M. (1989a). "Evaluation of surface and film diffusion coefficients for carbon adsorption." Water Res., 23(3), 267-273.

Traegner, U., and Suidan, M. (1989b). "Parameter evaluation for carbon adsorption." J. Environ. Eng., 115(1), 109-128.

Weber, W., and Crittenden, J. (1975). "MADAM I: A numeric method for design of adsorption systems." J. Water Pollut. Control Fed., 47(5), 924-940.

Weber, W., and Smith, E. (1986). "Removing dissolved organic contaminants from water." Environ. Sci. Technol., 20(10), 970-979.

Weber, W., and Smith, E. (1987). "Simulation and design models for adsorption processes.” Environ. Sci. Technol., 21(11), 1040-1050.

Wolborska, A. (1999). "External film control of the fixed bed adsorption." Chem. Eng. J., 73(2), 85-92.

Zeng, H., Arashiro, M., and Giammar, D. (2008). "Effects of water chemistry and flow rate on arsenate removal by adsorption to an iron oxide-based sorbent." Water Res., 42(18), 4629-4636.

Zhang, R., and Ritter, J. (1997). "New approximate model for nonlinear adsorption and diffusion in a single particle." Chem. Eng. Sci. 52(18), 3161-3172. 